\title{
The Effect and Safety of Intravitrealranibizumab Combined with Vitrectomy for Proliferative Diabetic Retinopathy Treatment \\ H Rong ${ }^{1}, \mathrm{Y} \mathrm{Xu}^{1}$, M Zhang ${ }^{1}, \mathrm{M} \mathrm{Cai}^{1}, \mathrm{H} \mathrm{Cui}^{1}$
}

\begin{abstract}
Objective: In this paper, we aimed to evaluate the effect and safety of intravitrealranibizumab with vitrectomy for proliferative diabetic retinopathy (PDR) patients' treatment.

Methods: Total 26 PDR (29 eyes) patients admitted to our hospital between June 1, 2014 and January 1, 2015 were retrospectively analyzed. There were 13 cases (15 eyes) in vitrectomy (PPV) group and 13 cases in intravitrealranibizumab with vitrectomy (IVR/PPV) group. The surgery duration, electrocoagulationutilization, BCVA and complications were evaluated in the two groups.

Results: The mean surgery duration was significantly shorter in IVR/PPV group $(80 \pm 15.08 \mathrm{~min})$ than PPV group $(108.07 \pm 11.63 \mathrm{~min})(\mathrm{t}=5.822, \mathrm{P}=0.0000)$. The incidence of iatrogenicslit pore, vitreous hemorrhage, hyphema was significantly reduced in IVR/PPV group $(\mathrm{P}<0.05)$. At 6 month after operation, the visual acuity of patients were improved in both two groups and visual acuity increase of patients in IVR/PPV group was higher than PPV group $(\mathrm{P}=0.015)$.

Conclusion: Intravitrealranibizumab combined with vitrectomy for PDR patients' treatment significantly reduced the surgery duration, the incidence of bleeding and complications. It may be a promising therapy for PDR patients.
\end{abstract}

Keywords: Intravitreal ranibizumab, proliferative diabetic, retinopathy, vitrectomy, .

From: Department of Ophthalmology, The affiliated East Hospital of Tongji University, Pudong, Shanghai 200120, China.

Correspondence: Dr H Cui, Department of Ophthalmology, The Affiliated East Hospital of Tongji University, Pudong, Shanghai 200120, China, Fax: +86 021-61569781, e-mail: hongpingcui1@126.com 


\section{INTRODUCTION}

Proliferative diabetic retinopathy (PDR) is a serious blinding disease, which is characterized by vitreous hemorrhage, neovascularization originating from the retina and tractive retinal detachment. Pars plana vitrectomy (PPV) is usually reserved for PDR treatment. However, the PPV shows difficulties in the operation process. Hemorrhage during operation increases the difficulties by extending the operation time and increasing the risk of bleeding post-operation (1). Furthermore, the bleeding out of the control in surgery may result in operation failure.

Recently, advances have been achieved in exploring the mechanism of DR with the application of molecular biology and cell biology technique. Drugs targeting vitreous concentration of vascular endothelial growth factor (VEGF) rises significantly(2) and provides novel perspective for PDR treatment. Ranibizumab (Lucentis) is a recombinant, humanized, monoclonal antibody Fab that can inhibit the vascular proliferation by targeting VEGF-A. Intravitreal ranibizumab was firstly used for the treatment of neovascular age-related macular degeneration. The efficacy and safety of intravitreal ranibizumab treatment have been widely validated. It is reported that ranibizumab shows promising ambient effect in the perioperative period of PDR, but the long-term safety remained to be evaluated. For the ambient effect, ranibizumab has been widely used in vitreous body related surgery in clinic.

In this paper, we retrospectively analyzed the 26 PDR patients (29 eyes) admitted to our hospital. The efficacy of vitrectomy combined with intravitreal ranibizumab for PDR patients was evaluated, compared with vitrectomy surgery. 


\section{SUBJECTS AND METHODS}

\section{Patients}

Total 26 cases (29 eyes) diagnosed with proliferative diabetic retinopathy (PDR) between June 1, 2014 and January 1, 2015 were retrospectivelyanalyzed in our study. Patients with FBG (fasting blood-glucose) $\leq 7.8 \mathrm{mmol} / \mathrm{L}$ and diagnosed with Type II diabetes mellitus for more than 1 month were included in our work. The ultrasound B-mode scan showed that included patients presented withvitreous hemorrhage, exudative membraneand/ortraction retinal detachment (TRD). For PDR patients without vitreous hemorrhage, hyperplasia and/or TRD were tested by fundus examination. Best corrected visualacuity (BCVA) for patients was measured at light sense to 0.2. All the subjects were given vitrectomy treatment.

Patients conforming toexclusion criteria were excluded, such as (1) Patients with retinal artery occulusion, high myopia, pterygium, glaucoma, cornea disease and uveitis; (2) phacoscotasmus $\geq$ IV stage; (3) history of Glucocorticoid treatment within 6 months; (4) vitreous hemorrhage was not induced by PDR; (5) Patients with cardiovascular diseases (such as hypertension) and immune diseases (such as systematic lupus erythematosus, Behcet and Ankylosing Spondylitis).

\section{Groups}

The 26 cases were divided into two groups including vitrectomy (PPV) treatment group ( $\mathrm{n}=13 / 15$ eyes) and intravitrealranibizumab injection combined with vitrectomy (IVR/PPV) treatment group ( $\mathrm{n}=13 / 14$ eyes). There were 6 males ( 7 eyes) and 7 females ( 8 eyes) in PPV group. The mean age for the patients was $56.54 \pm 14.29$ years ranging from 32 to 82 years. The diabetesduration for patients ranged from 5 to 30 years and the mean duration was $13.31 \pm 7.57$ years. The preoperativeBCVA $(\log M A R)$ in cases of PPV group was between 0.7 and 2.0 and the 
mean BCVA was $\log \mathrm{MAR}(1.69 \pm 0.35)$, mean intraocular pressure $(\mathrm{IOP})=16.62 \pm 7.28 \mathrm{mmHg}(1$ $\mathrm{mmHg}=0.133 \mathrm{kPa})$.

Patients aging from 37 to 88 years (mean: $59.08 \pm 14.17$ years) were included in IVR/PPV group, which comprised 6 males ( 7 eyes) and 7 females ( 7 eyes). The baseline information for cases were mean diabetes duration $=13.85 \pm 7.80$ (range, $5 \sim 30$ ), mean preoperativeBCVA $(\operatorname{logMAR})=1.59 \pm 0.50($ range, $0.7 \sim 2.0)$, mean IOP pre-ranibizumab injection $=14.23 \pm 3.51 \mathrm{mmHg}$ and the mean IOP pre-vitrectomy was $13.69 \pm 2.42 \mathrm{mmHg}$. There was no significant difference in gender $\left(\chi^{2}=0\right)$, age $(\mathrm{t}=0.41)$, duration of diabetes $(\mathrm{t}=0.15)$ and preoperativeBCVA $(\mathrm{t}=0.47)$ between two groups. In addition, no difference was observedbetweenaverageIOP in PPV group and averageIOP pre-ranibizumab injection and pre-vitrectomy $(\mathrm{P}>0.05)$.

\section{Ranibizumabintravitreal injection}

The patients in IVR/PPV group were given the intravitreal injection of ranibizumab by one skilled physician at 3-7 d before vitrectomy surgery. Before treatment, tropicamide (10ml:50mg, SANTEN OY, JAPAN) was administered to the eyes of patients. Surface anesthesia was performed for patients by Proxymetacaine hydrochloride (15ml:75mg,s.a. ALCON-COUVREUR n.v., Belgium). Then, 5\% povidone-iodine was applied for preoperative disinfection ofthe conjunctival sac (500ml: 25mg, Shanghai likang disinfection technology co., LTD, Shanghai, China) with an eye speculum. After the conjunctival sac was washed with normal saline solution, we used forceps to stabilize the eye and made a location $3.5 \mathrm{~mm}$ behind limbus. The intravitreal injections of $0.5 \mathrm{mg}$ Ranibizumab $(0.2 \mathrm{ml}: 2 \mathrm{mg}$, Novartis Pharma Stein AG, Switzerland) were performed with a 4.5 -gauge needle. The needle was visualized inwall of eyeball about $1 \mathrm{~cm}$. After the needle was withdrawn, the needle mouth was pressed for a moment and dropped with 5\% Povidone-iodine solution, followed by washing with normal saline. Finally, the 
Rong et al

ofloxacin (3.5g: 10.5mg, Shenyang xing qi pharmaceutical co., LTD, Shenyang, China) was instilled in eyes.

\section{Vitrectomy surgery}

The vitrectomy surgery was performed by a singlesurgeon with the application of ALCON Accurus 800 vitreous cutting system and Novus Spectra high power 532nm laser system. The patients in both two groups were subjected to $23 \mathrm{G}$ pars plana vitrectomy with standard three-port system. Patients complicated with cataract also underwent the ultrasonic emulsification. The surgical procedures included eliminating vitreous hemorrhage, stripping new tunica vasculosalentis, releasing vitreous traction, returning the retina, a total gas-fluid exchange.

\section{Evaluation}

The duration of operation and the use of electrocoagulation was recorded. The complications such as iatrogenicslit pore, blooding in early period of postoperation (within 1 month), blooding in the later period (more than 1 month). BCVA was tested at 6 month after operation.

\section{Statistical analysis}

The statistical analysis was performed by SPSS18.0. All the data were expressed as mean \pm SD. Difference between groups were analyzed by $t$ test and Fisher exact test for measurement data and enumeration data, respectively. $\mathrm{P} \leq 0.05$ was considered to be significant.

\section{RESULTS}

\section{Surgery duration}

The mean surgery duration in IVR/PPV group was $80 \pm 15.08 \mathrm{~min}$, ranging from $105 \mathrm{~min}$ to 60 min, while the mean surgery duration in PPV group was 108.07 \pm 11.63 min (rang, 93 min-130 
$\min )$. There was significant difference in the surgery time between two groups $(\mathrm{t}=5.822, \mathrm{P}=0.000)$.

\section{BCVA post-operation}

In the follow-up period, the mean BCVA (logMAR) of IVR/PPV group was $0.87 \pm 0.50$, ranging from 0.3 to 1.7. In PPV group, the BCVA (logMAR) of patients were between 0.7 and 2.0 with mean of $1.37 \pm 0.47$. The mean BCVA in PPV group was significantly higher than IVR/PPV group $(\mathrm{t}=2.803, \mathrm{P}=0.015)($ Table 1$)$.

\section{Outcomes}

Total 10 patients (11 eyes) with cataract in IVR/PPV group were subjected to ultrasonic emulsification and 11 patients (12 eyes) in PPV group. The 8 eyes in IVR/PPV group were filled with silicone oil, while 15 eyes in PPV group was filled with silicone oil in the operation. In the IVR/PPV group, 1 eyewas subjected to electrocoagulation for excessive bleeding and 7 eyes in PPV group. Iatrogenicslit porewas observed in 1 eye of IVR/PPV group and 8 eyes in PPV group. In the early postoperative period, 2 eyes in IVR/PPV group suffered recurrent bleeding and 9 eyes in PPV group. In the later period after operation, no recurrent bleeding was observed in IVR/PPV group and total 6 eyes suffered recurrent bleeding in PPV group. The incidence of iatrogenicslit pore, bleeding, recurrent bleeding was significantly lower in IVR/PPV group, compared with PPV group $(\mathrm{P}<0.05)$. The frequency of the use of electrocoagulation was significantly decreased in IVR/PPV group, compared with PPV group ( $\mathrm{P}=0.035)$. Additionally, 3 eyes in PPV group suffered iris neovascular glaucoma within 6 month after operation. All the information was listed in Table 2.

\section{DISCUSSION}

PDR patients are usually companied with the destruction of retinal vessel, retinaischemia, 


\section{Rong et al}

appearance of retina and irisneovascularization that may result in vitreous hemorrhage and tractional retinaldetachment.During the vitrectomy surgery, bleeding is an event attributed to retinal neovascularization and vitreous hemorrhage, which may result in ambiguous operation view and increase the difficulties in surgery. Previous studies showed that retinal neovascularization is the main cause of hemorrhage during operation, which is mediated by the secretion of cytokines caused by retinaischemia. VEGF as the cytokine, plays a potent role in angiogenesis and tunica vasculosa lentis development (3). It is reported that VEGF is overexpressed in the progression and development of DR and is the main pathogenic factor for DR.

The current drugs targeting VEGF mainly include Avastin, Lucentis and Macugen(4). Lucentis is considered to be the most effect drug and has been approved to be the only drug targeting VEGF for treating the eye diseases by the USA FDA. The efficacy and safety of intravitreal ranibizumab have been proved in treating AMD by a large amount of studies (5).

In our work, 13 cases (14 eyes) in IVR/PPV group were pre-treated by intravitreal ranibizumab and then subjected to vitrectomy. The retina and iris neovascularization was regressed significantly, which obviously reduced the incidence of bleeding and iatrogenicslit pore, frequency of electrocoagulation utilization and surgery duration, compared with PPV group $(\mathrm{P}<0.05)$. Intravitreal ranibizumabsignificantly reduced the iatrogenic injury of retina and hemorrhage during operation and thus increased the probability of surgery success and reduced the incidence of recurrence.

Our data also showed that the incidence of bleeding pre- and post-operation was significantly reduced in IVR/PPV group compared with PPV group. We speculated that Ranibizumab played a key role in inhibiting the activation of VEGF-A by binding to VEGF-A1 and VEGF-A2 on the cell surface. Ranibizumabtreatment reduced the proliferation of vascular 
endothelial cell,blood vesselleakage and neovascularization.

In addition, the visual acuity of patients in both two groups were improved significantly after treatment $(\mathrm{P}<0.05)$, which suggested that vitrectomy improved the restoration of retina, and facilitate visual acuity increase.

The visual acuity of patients in IVR/PPV group increased significantly compared with PPV group, which might be due to the decreased complications by intravitrealranibizumab. Van Geest et al. (6) suggested that the treatment with drug targeting VEGF was closely associated with the imbalance of connective tissuegrowth factor and VEGF. The clinical study indicated that bevacizumabaggravated the fibrosis and could promote the hyperplasia development. Tolentino(7) reported that ranibizumab injection reduced the inflammation response in eyes, but induced the bleeding and stroke. Sharma et al (8) 10 reported that the inflammation response in eyes significantly inhibited by ranibizumab injection, compared with bevacizumab treatment. Although there were no serious complications in patients treated with ranibizumab, but ranibizumab injection increased the risk of thrombus of artery. In this paper, no adverse effect was observed in IVR/PPV group, which was consistent with the previous reports by Feiner et al.(9) and Bakri et al.(10). Our study was limited by the small size of samples and short follow-up period. Further studies should be conducted to validate the safety of intravitreal ranibizumab.

In conclusion, vitrectomy combined with intravitreal ranibizumab significantly improved the visual acuity and surgery success, and reduced the complications. However, the long-term safety and efficacy of intravitreal ranibizumab needed to be further validated. 


\section{REFERENCES}

1. Oyakawa RT, Schachat AP, Michels RG, Rice TA: Complications of vitreous surgery for diabetic retinopathy:I.Intraoperative complications. Ophthalmology. 1983; 90: 517-21.

2. Moradian S, Ahmadieh H, Malihi M, Soheilian M, Dehghan MH, Azarmina M: Intravitreal bevacizumab in active progressive proliferative diabetic retinopathy. Graefe's Archive for Clinical and Experimental Ophthalmology. 2008; 246: 1699-705.

3. Penn J, Madan A, Caldwell R, Bartoli M, Caldwell R, Hartnett M: Vascular endothelial growth factor in eye disease. Progress in retinal and eye research. 2008; 27: 331-71.

4. Wang H, Sun X, Liu K, Xu X: Intravitreal ranibizumab (lucentis) for the treatment of diabetic macular edema: a systematic review and meta-analysis of randomized clinical control trials. Current eye research. 2012; 37: 661-70.

5. Pieramici DJ, Avery RL: Ranibizumab: treatment in patients with neovascular age-related macular degeneration. 2006.

6. Van Geest RJ, Lesnik-Oberstein SY, Tan HS, Mura M, Goldschmeding R, Van Noorden CJ, Klaassen I, Schlingemann RO: A shift in the balance of vascular endothelial growth factor and connective tissue growth factor by bevacizumab causes the angiofibrotic switch in proliferative diabetic retinopathy. Brit J Ophthalmol 2012; 96: 587-90.

7. Tolentino M: Systemic and ocular safety of intravitreal anti-VEGF therapies for ocular neovascular disease. Survey of ophthalmology. 2011; 56: 95-113.

8. Sharma S, Johnson D, Abouammoh M, Hollands S, Brissette A: Rate of serious adverse effects in a series of bevacizumab and ranibizumab injections. Can J Ophthalmol 2012; 47: $275-9$.

9. Feiner L, Barr EE, Shui Y-B, Holekamp NM, BRANTLEY Jr MA: Safety of intravitreal 
injection of bevacizumab in rabbit eyes. Retina. 2006; 26: 882-8.

10. Bakri SJ, Cameron JD, McCannel CA, Pulido JS, Marler RJ: Absence of histologic retinal toxicity of intravitreal bevacizumab in a rabbit model. Am J Ophthalmol 2006; 142: $162-$ 4. 
Table 1: The improvement of BCVA in two groups

\begin{tabular}{lllll}
\hline Group & $\begin{array}{c}\text { BCVA (logMAR) } \\
\text { pre-operation }\end{array}$ & $\begin{array}{c}\text { BCVA (logMAR) } \\
\text { post-operation }\end{array}$ & $\mathbf{t}$ & $\mathbf{P}$ \\
\hline IVR/PPV & $1.59 \pm 0.50$ & $0.87 \pm 0.50$ & 4.77 & 0.000 \\
$\mathrm{PPV}$ & $1.69 \pm 0.35^{*}$ & $1.37 \pm 0.47 \#$ & 2.564 & 0.023 \\
\hline *P $>0.05$ compared with IVR/PPV group before operation; \#P $<0.05$ compared with IVR/PPV after \\
operation.
\end{tabular}

Table 2: The outcomes after treatment in two groups

Groups electrocoagulation iatrogenic slit pore bleeding ( $\leq 6$ month) bleeding ( $\geq 6$ month)

\begin{tabular}{lllll}
\hline IVR/PPV & $1(7.14)$ & $1(7.14)$ & $2(14.28)$ & $0(0)$ \\
PPV & $7(46.67)^{*}$ & $8(53.33) *$ & $9(60) *$ & $6(40) *$ \\
\hline$* \mathrm{P}<0.05$ compared with IVR/PPV group.
\end{tabular}

